\title{
Structural Realism: Continuity and its Limits
}

To appear in A. Bokulich and P. Bokulich (eds.): Scientific

Structuralism (Boston Studies in the Philosophy of Science)

\author{
Ioannis Votsis \\ University of Duesseldorf \\ votsis@phil-fak.uni-duesseldorf.de
}

\begin{abstract}
Structural realists of nearly all stripes endorse the structural continuity claim. Roughly speaking, this is the claim that the structure of successful scientific theories survives theory change because it has latched on to the structure of the world. In this paper I elaborate, elucidate and modify the structural continuity claim and its associated argument. I do so without presupposing a particular conception of structure that favours this or that kind of structural realism. Instead I focus on how structural realists can best account for the neutrally formulated historical facts. The result, I hope, crystallises some of the shared commitments, desiderata and limits of structural realists.
\end{abstract}

\section{Introduction}

Structural realism comes in various shapes and sizes. First there is the epistemic kind which holds that at best we can have knowledge of the structure of the world. This comes in two main flavours: à la Ramsey (e.g. John Worrall and Elie Zahar 2001) claiming that the structure of the world is reflected in the Ramsey sentence of successful scientific theories and à la Russell (e.g. Ioannis Votsis 2005) claiming that we can infer certain things about the structure of the world from the structure of our perceptions. Then there is the ontic kind which also comes in a multitude of flavours, three of which stand out: (i) the 'no objects view' (e.g. James Ladyman 1998) according to which there exist no objects only structures, (ii) the 'no individuals view' (e.g. Steven French and Decio Krause 2006) which maintains that there exist no individuals but only non-individual objects and structures and (iii) the 'no intrinsic natures view' (e.g. Ladyman 2007) which eliminates intrinsic natures in favour of haecceity-free individuals and structures. ${ }^{1}$ Finally, there is the methodological kind which concentrates on the role shared structure plays in characterising scientific theories, in relating high-level theory to low-level data and in identifying links between predecessor and successor theories (see Katherine Brading and Elaine Landry 2006).

That the different kinds of structural realism share less than their name suggests is something that is increasingly becoming apparent. ${ }^{2}$ One major disagreement relates to the way structure gets demarcated. It is not merely a question of which formal tools are best equipped for the job, e.g. Ramseyfication, set theory, group-theory, category theory, etc. It is also a question of how we draw the line between the structural and the non-structural. For example, some circumscribe the structural so as to include

\footnotetext{
${ }^{1}$ Concerning the 'no objects view', Ladyman insists he never intended to say that no objects exist. He admits, however, that certain of his early pronouncements have contributed to this misinterpretation.

${ }^{2}$ In a recent workshop on structural realism organised in Banff by Elaine Landry, Ladyman urged the participants, who parenthetically represented almost the whole spectrum of different structural realist positions and included most of the main players, to find a mutually agreeable formulation of what is common to all. What became clear by the end of the workshop was that no such formulation can easily be produced.
} 
structural information about intrinsic properties (e.g. Votsis), while others deny the very existence of intrinsic properties (e.g. Ladyman).

Disagreements aside, all structural realists (bar perhaps the methodological ones) appropriate the preservation of structure through historical theory change as evidence for their respective views. They thus endorse what I will henceforth call 'the structural continuity claim'. Roughly put, this is the claim that the structure of successful scientific theories survives theory change in virtue of the fact that it correctly reflects structural features of the world. In other words, structures are preserved through theory change on account of their truth or approximate truth - hereafter designated by '(approximate) truth'.

The structural continuity claim makes its debut appearance in Henri Poincaré's Science and Hypothesis. As John Worrall (1989) first pointed out, Poincaré utilises the structural continuity claim to motivate a version of epistemic structural realism. ${ }^{3}$ Poincaré argues that only structural features of theories survive theory change and the reason for their survival is that they have somehow latched on to the structure of the world. He cites the preservation of Augustin-Jean Fresnel's equations in James Clerk Maxwell's electromagnetic theory as evidence for that claim. The equations successfully describe the reflection and refraction of light when it is passing through media of different refractive indices. Under Fresnel's interpretation, light consists of vibrations that are transmitted through the ether, a ubiquitous yet virtually imperceptible material medium. Of crucial importance is the fact that Fresnel's interpretation of the nature of light is not necessary for the empirical exploitation of Maxwell's equations and, a fortiori, not necessary for the empirical exploitation of Fresnel's own equations since the latter can be derived from the former. It is no wonder then that the interpretation is made redundant in Maxwell's mature electromagnetic theory. This is taken by Poincaré and subsequent structural realists to mean that Fresnel's (and Maxwell's) equations provide at most a structural account of light.

Let us now reconstruct the argument whose conclusion is the more polished structural continuity claim:

1. Only structural elements of predictively and explanatorily successful scientific theories have been (and will be) preserved through theory change.

2. Preservation of an element implies its (approximate) truth.

3. Non-preservation of an element implies its (approximate) falsity.

$\therefore$ The preservation of structural elements of predictively and explanatorily successful scientific theories through theory change implies their (approximate) truth. The non-preservation of non-structural elements implies their (approximate) falsity.

A few qualifications are in order. First, by 'elements' I mean statements about the world that have a truth value. Structural elements are truth-valued statements whose content is purely structural. Second, for most structural realists this 'purely structural' clause concerns only the unobservables. What counts as an unobservable differs in

\footnotetext{
${ }^{3}$ The structural continuity claim is not the sole motivation for Poincaré's epistemic structural realism as I indicate in Votsis (2004, ch. 2).
} 
some of these accounts. Third, since different conceptions of structure may lead to different formulations of this argument I have formulated the argument in the most general way possible, i.e. without specifying where to draw the line between structure and non-structure. After all, structural realists of different stripes agree on the relevant historical facts when they are neutrally stated, e.g. that a set of equations belonging to some past theory is derivable from its successor. Their disagreement lies only in the interpretation of such facts as evidence for their specific brand of structural realism.

In this paper I aim to elucidate, improve and extend the structural continuity claim and its associated argument. In so doing, I will not presuppose a particular conception of structure that favours this or that kind of structural realism but will instead focus on how structural realists can best handle the neutrally formulated historical facts. A positive consequence of this approach is that the results will be pertinent to both epistemic and ontic structural realists. A negative consequence is that various significant issues, such as how best to draw the structure vs. non-structure distinction as well as whether the distinction is even feasible (questioned, for example, in Bas van Fraassen 2006, p. 290 and in Stathis Psillos 1999, p. 157) will be left untouched. ${ }^{4}$ My intended audience is therefore those who at least provisionally accept that the structure vs. non-structure distinction can be drawn but are unclear about the details those that are independent of the particular form of the distinction - of the structural continuity claim and its associated argument.

\section{Not all Structures are Preserved}

Not all structures are created equal. Some play no active role in the predictive and explanatory success of a theory because they do not correspond to any structure in the world. Their non-preservation does not therefore encumber the structural realist. Traditional scientific realists have long employed a distinction between essential and idle posits to weed out those elements of theories that played no substantial role in their predictive and explanatory success. An analogous distinction is required for the structural realists. Henceforth I will brand operative those structures that are responsible for a theory's genuine predictive and explanatory success. Those that fail this condition, I will brand inoperative.

Under the foregoing characterisation Fresnel's equations count as operative structural elements, for they are arguably the sole purveyors of the success enjoyed by Fresnel's theory of light. More examples of operative structural elements will be discussed in the sections below. For now let us consider an example of an inoperative structural element. Such examples are plentiful in the history of science. After all, most conjectures are likely to be predictively and explanatorily unsuccessful. ${ }^{5}$ Take August Weismann's claim that different cells contain different components of hereditary material and are distributed to different parts of an organism's body so as to locally oversee that part's development. In his bid to defeat structural realism, Kyle Stanford (2006, p. 181) offers Weismann's claim as an example of a structural element from biology that did not survive theory change. Contra Stanford, we can simply point out that Weismann's claim did not enjoy genuine predictive success. Thus Weismann's claim is not merely a structural element that did not survive theory change but also an

\footnotetext{
${ }^{4}$ I have defended a version of the structure vs. non-structure distinction in Votsis (2007).

${ }^{5}$ Construing such conjectures structurally does nothing to reverse this unsuccessfulness.
} 
inoperative element. For this reason its abandonment does not threaten the structural realist.

Modified accordingly, premise one now reads as follows.

1a. All and only operative structural elements of scientific theories have been (and will be) preserved through theory change.

Two provisos need to be made here. First, the clause 'predictively and explanatorily successful' is now packaged inside the concept operative. Second, the reformulation makes clear that not only are all predictively and explanatorily successful elements that survive structures, but also that all predictively and explanatorily successful structures survive.

\section{Not all Preserved Structures are Intact}

As many authors have rightly pointed out the neat preservation of structure exhibited by the Fresnel-Maxwell case is atypical in the history of science (e.g. Michael Redhead 2001). ${ }^{6}$ More often a structure belonging to a superseded theory can be recovered only as a limiting case of a successor theory's structure. Aware of this, Worrall (1989) reasoned that structural realism benefits from 'limiting case' survival when appeal is made to the correspondence principle. According to Heinz Post's formulation, "this is the requirement that any acceptable new theory L should account for its predecessor S by 'degenerating' into that theory under those conditions under which S has been well confirmed by tests" (1971, p. 228). Worrall notes that given that the principle operates solely on the mathematical level its applicability "is not evidence for full-blown realism - but, instead, only for structural realism" (1989, p. 161).

A refinement of premise one that takes into consideration the need to employ the correspondence principle takes the following form:

1b. All and only operative structural elements of scientific theories have been (and will be) preserved through theory change either intact by derivation or suitably modified in accordance with the correspondence principle.

Worrall's remarks on the link between the correspondence principle and structural realism are rather brief and suggestive. Luckily, Redhead has made some progress on this front.

Consider a one-parameter family of structures $\left\{S_{p}\right\}$ where the parameter $p$ is a continuously variable real number. Let us suppose for values of $p$ unequal to zero the structures $S_{p}$ are all qualitatively the same, as $p$ varies the structure changes, but in a continuous way. But suppose the change in structure suffers a discontinuity at the point $\mathrm{p}=0, \mathrm{~S}_{0}$ is qualitatively distinct from all the $\mathrm{S}_{\mathrm{p}}$ with $\mathrm{p} \neq 0$. We may say that the family of structures is stable for $\mathrm{p} \neq 0$, but exhibits a singularity at $\mathrm{p}=0$ (ibid., p. 86) [original emphasis].

\footnotetext{
${ }^{6}$ It is atypical but not unique. Several structures postulated within the framework of the caloric theory of heat have survived the theory's demise and are still with us today, e.g. Sadi Carnot's principle of maximum efficiency.
} 
Redhead thus identifies two kinds of structure transformations: continuous and discontinuous. ${ }^{7}$ Whether or not a structure transformation is discontinuous depends on what makes a structure the kind of structure it is, i.e. what we deem to be its essential (read: defining) features. Redhead offers an instructive example from geometry. Think of transforming a circle on a Euclidean plane into any closed curve. Suppose the essential features in this case are the following: (a) that the shape completely encloses an area and (b) that it has no endpoints. Since these two conditions are essential features of closed curves (including circles), the transformation is continuous with respect to the relevant group of homeomorphisms. Contrast this with the transformation of any closed curve (including a circle) into a straight line. In this case (a) and (b) are lost and so the transformation qualifies as discontinuous.

In his example Redhead neglects to highlight that some continuous transformations can easily be turned into discontinuous ones if the appropriate essential features are available. Think of the circle-to-closed curve transformation again. One of the defining characteristics of circles is that they possess a unique point equidistant from the set of points that bounds them, i.e. a centre. Modulo this essential feature the aforementioned transformation becomes discontinuous as no other closed curves share this feature with circles.

As it stands, the notion of discontinuous transformation fails to do justice to the varying degrees of discontinuity. For example, there is a clear sense in which the circle-to-closed curve transformation is less discontinuous than the circle-to-straight line transformation. To redress this issue we need to establish a more fine-grained account of discontinuous transformations. A first step in the right direction is to divide the original notion into two notions: 'partially discontinuous' and 'fully discontinuous'. The first notion applies when the transformation brings about the loss of some but not all of the essential features that a structure possesses. In general, the less essential features lost the more continuous the transformation. The second notion applies when all essential features are lost. Changes of this magnitude make it difficult, if not impossible, to claim that successor structures have a non-accidental kinship to predecessor structures. ${ }^{8}$ Although refinements to these notions and perhaps even additional notions are required to deal with further problems, e.g. some essential features may be more essential than others and hence will need to be differentially weighted, the two notions will do for the purposes of this paper.

At this point it is worth asking how the different kinds of structure transformations fit into the puzzle of relating old and new structures. The simple answer is that they are either all exemplified in the history of science or they could be so exemplified. Since discontinuous transformations are quite prevalent in modern physics and indeed more challenging to legitimate as genuine cases of substantial (albeit partial) continuity I will focus the discussion on them. To explain the rationale behind such transformations, imagine, as a first approximation, a successor structure as typically possessing one or more additional parameters than its predecessor. We can think of the predecessor structure as a more abstract, less approximately true, more idealised

\footnotetext{
${ }^{7}$ Though not a structural realist, Robert Batterman (2002, pp. 17-19) draws a similar distinction between reduction (where the limit is regular) and intertheoretic relations (where it is singular).

${ }^{8}$ It may still be possible that two structures are somehow partially continuous on the basis of nondefining features. I mention this only as food for thought as I do not really put much trust in the claim that continuity of this kind is sufficient for (structural) realist purposes.
} 
version of the successor structure (e.g. Wladyslaw Krajewski 1977). Neutralising these parameters from the successor structure thereby allows us to recover the predecessor one. In the above framework, the neutralisation of a parameter is achieved by suitably modifying its value, e.g. by setting it to zero. Assuming, as it seems we must, that the parameter at issue corresponds to an essential feature of the successor structure entails that neutralising it amounts to the removal of that feature and hence to a discontinuous transformation. In cases where some essential features survive the transformation we can speak of partial continuity or discontinuity. When all essential features are lost we can speak of full discontinuity. Only fully discontinuous transformations are undesirable for the task of supporting structural realism.

We are now in a position to unveil a bespoke correspondence relation for the structural realist:

A structure $S^{\prime}$ and its predecessor structure $S$ correspond if and only if with respect to a given parameter class there is a transformation from $S^{\prime}$ to $S$ that is either (a) continuous or (b) partially discontinuous.

It is no doubt time for an example. Redhead cites the relation between Minkowskian and Galilean space-times as a prominent example of a discontinuous transformation. What kind of a discontinuous transformation is it? One of the essential features of Minkowski space-time is that it allows for a non-singular metric which is represented by the matrix diagonal $\left(1,-1 / c^{2},-1 / c^{2},-1 / c^{2}\right)$, where $c$ is the speed of light in a vacuum. Since the metric is non-singular, the above matrix diagonal has an inverse, namely $\left(1,-c^{2},-c^{2},-c^{2}\right)$. If we let $c=\infty, 1 / c=0$ and the metric becomes singular, since the corresponding matrix diagonal $(1,0,0,0)$ allows no inverse. By doing so, relativity of simultaneity disappears and we recover Galilean space-time. The fact that several essential features of Minkowski space-time survive the transformation, e.g. the structure still forbids absolute velocity and absolute spatial separation, entails that the transformation is only partially discontinuous. Thus the relation between Minkowski and Galilean space-times counts as evidence for the structural continuity claim as the transformation preserves some essential features in its wake.

In light of our discussion of discontinuous transformations, I suggest that we modify the first premise thus:

1c. All and only operative structural elements of scientific theories have been (and will be) preserved through theory change either intact by derivation or via a transformation from new to old structure that is either (i) continuous or (ii) partially discontinuous.

What makes discontinuous transformations capable of supporting the structural continuity claim? Astonishingly, one finds little by way of argument in Redhead's otherwise fecund paper. He resorts to metaphorical language, claiming that, if, like the mathematician, we see how natural the leap is to introduce or remove a feature from a structure, then we realise that discontinuous transformations of structures in physics are cases of structure preservation (ibid., p. 88). Discontinuous transformations must be put on firmer footing than this. I have already intimated how this may be done. The introduction of the two notions of discontinuous transformation brings out the fact 
some transformations are unreservedly radical while others less so. Surely the latter are capable of supporting the structural continuity claim for they display constancy with respect to some essential features between old and new structures. To stress this point in a different way, just think how improbable it would be that any two structures accidentally happen to be connected via partially discontinuous transformations. To test this, take an algorithm that generates (pseudo-) random pairs of structures. Because a great many structures share no essential features at all, the odds of getting a pair that corresponds via partially discontinuous transformations are very small.

\section{Not all Structures have Predecessors}

Not all successor equations have limiting case analogues in the predecessor theory. Hans Radder (1996) cites the relativistic equation $E=m_{0} c^{2}$ for a particle's energy with rest mass $m_{0}$. No analogue of it exists in classical mechanics so any talk of structure transformation from new to old theory would be pointless. Radder and other philosophers tout this fact as detrimental to the correspondence principle. Since the structural continuity claim banks on the principle, the objection threatens to derail structural realism itself.

A more careful look at the correspondence principle reveals how remarkably easy it is to answer this objection. It is merely a matter of revealing how the objection confounds the scope of the principle. The principle does not require that all (successful) successor structures correspond to (successful) predecessor structures. Let's not forget that in the (structural) realist's eyes successor theories will venture beyond their predecessors, describing and predicting new classes of phenomena with the help of completely new structures. What the principle requires is that all (successful) predecessor structures correspond to (successful) successor structures. As such the objection leaves the correspondence principle unfazed.

\section{Kuhn Loss}

The term 'Kuhn loss' seems to have been coined by Post (ibid.). He quotes a relevant passage from Kuhn who says "new paradigms seldom or never possess all the capabilities of their predecessors" ([1962]1996, p. 169). What does Kuhn mean by capabilities? His scattered thoughts on the matter seem to mostly point to the capability of explaining phenomena. For example he speaks of the loss of such capabilities in terms of the new paradigm being deprived of "some actual and much potential explanatory power" and of its "failure to explain" ([1962]1996, p. 107). If such losses exist, they seem to undermine the realist claim that successor theories incorporate all of the successes of their predecessors and hence are strictly more approximately true than their predecessors. How does this affect the structural continuity claim? Suppose such losses were operative structural elements. Under this supposition it would no longer be true that all operative structural elements survive theory change and therefore the structural continuity claim would be false.

There are two main readings of Kuhn's view. According to the narrow reading, offered by Post, a Kuhn loss is the "loss of successful explanatory power" (ibid., p. 229) [emphasis added]. Post goes on to clarify that Kuhn-losses are those wellconfirmed parts of a superseded theory that were not saved in its successor and rejects that any such losses ever occur (p. 230). By contrast, Alexander Bird's interpretation of Kuhn's view is more relaxed, requiring only that a phenomenon "in an earlier period was held to be successfully explained" (2004) [emphasis added]. As we shall 
shortly see the controversy over the occurrence of Kuhn-losses hinges on how widely one reads the loss of explanatory power.

Let us first consider the wide notion of Kuhn loss. Thus defined the notion has various historical instantiations. A frequently discussed example concerns the loss by Newtonians of the Cartesian ability to explain why "the planets lie in approximately the same plane" and why "planets orbit the sun in the same direction [and indeed in the same direction as the Sun's spin]" (James McAllister 2007, p. 18). ${ }^{9}$ According to this explanation the planets and any other celestial objects for that matter (including comets) are kept in orbit around a star by hitching a ride on the same fluid vortex. As the vortex turns only in one direction so do the objects that ride on it.

The explanation was certainly 'held to be successful' by some and hence qualifies as an instantiation of the wide notion of Kuhn loss. It does not, however, qualify as an instantiation of the narrow notion since the explanation was never well-confirmed. Over hundreds of years no such thing as a fluid vortex has ever been detected. Moreover, there are positive reasons to reject the Cartesian explanation because it does not account for the following anomalies. Various objects in our solar system, e.g. Neptune's moon Triton as well as comet Halley, travel in the opposite direction to the Sun's spin. The same irregularities seem to hold for solar systems other than our own. A recently discovered exoplanet (WASP-17b) is the first known to travel against its star's rotation. ${ }^{10}$ That the orbit of objects in a solar system all lie in approximately the same plane is also falsified by the existence of objects like the dwarf planet Pluto whose orbit is highly inclined.

The above problems clearly illustrate that the Cartesian explanation was never a serious contender. We still do not have a well-confirmed explanation regarding the orbits of objects around stars. ${ }^{11}$ Realists (structural or otherwise) need explanations, but not those lacking robust empirical merits. In sum, although the Cartesian explanation qualifies as a Kuhn loss, under the wide construal of the notion, it is not the kind of loss that could challenge the cumulativity of scientific knowledge, or, in the case at hand, the structural continuity claim.

Let us then consider the narrow notion of Kuhn loss, i.e. the one that demands genuine empirical success from the lost ability. Despite all the commotion surrounding Kuhn loss, finding examples that satisfy this stronger notion is not an easy task. Radder (ibid., p. 63) puts forth Poiseuille's law as one - the only one it seems - such example. The law $Q=\pi r^{4} P / 8 \eta L$ determines an (nearly) incompressible fluid's rate of laminar flow $Q$ along a tube as a relation between the following quantities: the fluid's viscosity $\eta$, the radius $r$ and length $L$ of the tube and the pressure difference between the tube's two ends $P$. The law is arguably a structural element, as it requires no specific ontological interpretation of the involved quantities. It is also an operative element since it has been used to provide explanations and accurate quantitative predictions in a number of different domains including medicine where it is used to calculate blood flow. Crucially, and according to Radder, it is impossible to reproduce this law from quantum mechanical accounts of fluids. It thus

\footnotetext{
${ }^{9}$ For a similar point see also Paul Hoyningen-Huene (1993, p. 261).

${ }^{10}$ It is worth noting that we currently have evidence for the orbits of only around a dozen exoplanets.

${ }^{11} \mathrm{We}$ only have a tentative account in the guise of the nebular hypothesis which provides sketches of the formation and evolution of solar systems.
} 
seems to be a bona fide case of Kuhn loss in the narrow sense, threatening to undo the structural continuity claim.

Alas for Radder, one plain fact has been neglected. Poiseuille's law was never abandoned! It is in use today and can be found in numerous scientific textbooks. If it is still in use, then it was never lost, i.e. it is not a Kuhn loss. Having said this, Poiseuille's law presents another problem for (structural) realism. Up till now we have required that old structures be suitably preserved in new structures. Poiseuille's law is preserved but independently of any new structure. This contradicts what we required previously, namely that all successful predecessor structures have corresponding successor structures.

Despite appearances, the game is not lost for the (structural) realist. Some realists will no doubt argue that Poiseuille's law will eventually be derived from quantum mechanics. Bar that prospect, I want to maintain that there is nothing dire about the independent survival of a predictively and explanatorily successful structure. The structural continuity claim merely needs to be amended. New paradigms, theories or structures need not replace old ones in toto. That is, they need not range over all the old domains of phenomena, though we certainly expect them to unify a substantial chunk of the old domains with new domains of phenomena. So long as the unaccounted for domains are preserved nothing is really lost. Designations like 'the successor' are therefore clearly hyperbolic. The same point can be demonstrated in a much simpler way by reminding oneself of the fact that there exist two successors to the Newtonian paradigm, i.e. relativity theory and quantum mechanics.

This brings us to the final qualification of premise one.

1d. All and only operative structural elements of scientific theories have been (and will be) preserved through theory change either (a) intact by derivation or (b) via a transformation from new to old structure that is either (i) continuous or (ii) partially discontinuous or (c) intact but independent of any currently accepted structures.

\section{Inferences from Preservation}

Premises two and three of the structural continuity argument add up to the following claim: The preservation of an element is a necessary and sufficient condition of its (approximate) truth. ${ }^{12}$ No realist, I hope, ought to be happy to adopt such a strong claim. The preservation of an element through theory change is neither a necessary nor a sufficient condition for its (approximate) truth.

It is not a necessary condition because the preservation of $a(n)$ (approximately) true element is not guaranteed. An element might be cast aside because it is, or at least it seems to be, incompatible with certain parts of other theories. Perhaps instruments capable of assessing its empirical merits have not yet been invented. Even worse, it might be that no instrument capable for this assessment can be constructed. ${ }^{13}$ Thus an (approximately) true element may find itself thrown into the wastebasket of history.

\footnotetext{
${ }^{12}$ That the third premise amounts to preservation being a necessary condition of an element's (approximate) truth is more clearly seen when formulated in its contrapositive form, i.e. the (approximate) truth of an element implies its preservation.

${ }^{13}$ This last scenario finds support in some interpretations of the measurement problem in quantum mechanics.
} 
Kuhn losses, in the narrow construal of the concept, are therefore genuine possibilities.

Far from being outlandish, the necessity condition's failure can be witnessed in the actual historical record. Take the central claim of the kinetic theory of heat. The idea that heat is due to the motion of particles can be traced back to antiquity. It thereafter vanished only to reappear in the sixteenth century. Francis Bacon famously remarked that 'heat itself, its essence and its quiddity, is motion and nothing else'. Yet it was not until the nineteenth century when the work of bold experimentalists like Count Rumford, Humphry Davy and James Prescott Joule as well as the advent of new ideas like energy conservation allowed the successful development and rise to dominance of the kinetic theory of heat. To those itching to point out that the kinetic theory's central claim did eventually survive, it is worth reminding that premise three is tenseless. In other words, one should be able to apply the inference at any historical period, including the period between antiquity and the sixteenth century and not merely from the sixteenth century onward. It is also worth reminding that we could not be talking about a specific (approximately) true element that did not survive (during some period) unless that element did in due course survive. Needless to say that some (approximately) true elements may never be rediscovered.

Preservation is not a sufficient condition because the mere survival of a given element does not guarantee its (approximate) truth. This point has also been made by Hasok Chang (2003), though for reasons that do not exactly coincide with mine. Preservation does not guarantee (approximate) truth because it might simply be a by-product of the conservativeness of scientific theorizing. A well-document aspect of this conservativeness is our penchant for anthropomorphic, anthropocentric and teleological explanations. Thus for a long time it was natural to suppose the truth of the principle that an external force is required to keep things in motion. Our trust in this principle, as is well known, was withdrawn as a consequence of our acceptance of the law of inertia.

A strict preservationist will no doubt protest against both my necessity and sufficiency objections. Had the scientific community been able to test the elements at issue sooner, the preservationist will insist, they would have surely uncovered their empirical merits or lack thereof. Thus (approximately) true elements would be duly preserved and (approximately) false ones duly abandoned. Though this statement is largely correct, notice that now the empirical merits of elements take centre stage, not their state of preservation. In a nutshell, the issue of preservation becomes parasitic on the issue of empirical merits.

It has not been my intention to dismiss preservation as a hopeless concept but rather to shed light on its scope and the origin of its strength. It is still true after all, especially in more recent times, that preservation and (approximate) truth are substantially correlated. This correlation can be explained by the fact that scientists are now more likely than they once were to preserve those components that have empirical merits. It is for that reason highly unlikely that narrowly construed Kuhn 
losses will be found in abundance. ${ }^{14}$ This brings us to the final modification of the structural continuity argument.

1e. Approximately all and only operative structural elements of scientific theories have been (and will be) preserved through theory change either (a) intact by derivation, or (b) via a transformation from new to old structure that is either (i) continuous or (ii) partially discontinuous or (c) intact but independent of any currently accepted structures.

2a. Preservation is a reliable guide to (approximate) truth.

3a. Non-preservation is a reliable guide to (approximate) falsity.

$\therefore$ The preservation of approximately all and only operative structural elements of scientific theories through theory change either via (a), (b) or (c) is a reliable guide to their (approximate) truth. The non-preservation of nonstructural elements and inoperative structural elements is a reliable guide to their (approximate) falsity.

\section{Conclusion}

I do not expect what has been said above to be the final word on these matters. The more one studies the history of science the more one finds cases that deserve special attention. This in turn translates into amendments of the premises of the structural continuity argument and ultimately amendments of the structural continuity claim itself. These amendments will probably continue the tendency of relaxing the link between preservation and (approximate) truth. For this reason it is perhaps better to think of the structural continuity argument as inductively strong rather than as deductive. I hope that this essay has laid the foundation for a more focused debate on the shared commitments, desiderata and limits of structural realists.

Acknowledgements: This work was in part made possible by funding from the German Research Foundation (Deutsche Forschungsgemeinschaft).

\section{References:}

Batterman, Robert (2002), The Devil in the Details: Asymptotic Reasoning in Explanation, Reduction, and Emergence, New York: Oxford University Press.

Bird, Alexander (2004), 'Thomas Kuhn', The Stanford Encyclopedia of Philosophy, Edward N. Zalta (ed.), URL =http://plato.stanford.edu/entries/thomas-kuhn/

Brading, Katherine and Elaine Landry (2006), 'Scientific Structuralism: Presentation and Representation', Philosophy of Science, vol. 73(5):571-581.

Chang, Hasok (2003), 'Preservative Realism and Its Discontents: Revisiting Caloric', Philosophy of Science, vol. 70(5): 902-912.

French, Steven and Harmke Kamminga (1993), Correspondence, Invariance and Heuristics: Essays in Honour of Heinz Post, Boston Studies in the Philosophy of Science, vol. 148, Dordrecht: Kluwer Academic Press.

French, Steven and Decio Krause (2006), Identity in Physics: A Historical, Philosophical and Formal Analysis, Oxford: Oxford University Press.

Hoyningen-Huene, Paul (1993), Reconstructing Scientific Revolutions, Chicago: University of Chicago Press.

\footnotetext{
${ }^{14}$ Under the current qualification, isolated incidents of narrowly construed Kuhn losses are not sufficient to undermine the structural continuity claim.
} 
Krajewski, Wladyslaw (1977), Correspondence Principle and Growth of Science, Dordrecht: D. Reidel Pub. Co.

Kuhn, Thomas ([1962]1996), The Structure of Scientific Revolutions, $3^{\text {rd }}$ edition, Chicago: University of Chicago Press.

Ladyman, James (1998), 'What is Structural Realism?', Studies in History and Philosophy of Science, vol. 29: 409-424.

- (2007), 'Structural Realism', The Stanford Encyclopedia of Philosophy, Edward N. Zalta (ed.), URL =http://plato.stanford.edu/entries/structural-realism/

McAllister, James (2007), 'Introduction. Dilemmas in Science: What, Why, and How' in Knowledge in Ferment, Leiden: Leiden University Press.

Poincaré, Henri ([1905]1952), Science and Hypothesis, New York: Dover.

Post, Heinz R. (1971), 'Correspondence, Invariance and Heuristics', repr. in S. French and H. Kamminga (eds.), Correspondence, Invariance and Heuristics: Essays in Honour of Heinz Post, Boston Studies in the Philosophy of Science, vol. 148, Dordrecht: Kluwer Academic Press, 1993.

Psillos, Stathis (1999), Scientific Realism: How Science Tracks Truth, London: Routledge.

Radder, Hans (1996), In and about the World: Philosophical Studies of Science and Technology, New York: S.U.N.Y. Press.

Redhead, Michael L.G. (2001), 'The Intelligibility of the Universe', in A.O'Hear (ed.) Philosophy at the New Millennium, Cambridge: Cambridge University Press.

Stanford, Kyle (2006), Exceeding Our Grasp: Science, History, and the Problem of Unconceived Alternatives, Oxford: Oxford University Press

Van Fraassen, Bas C. (2006), 'Structure: Its Shadow and Substance', British Journal for the Philosophy of Science, vol. 57(2): 275-307.

Votsis, Ioannis (2004), The Epistemological Status of Scientific Theories: An Investigation of the Structural Realist Account, London School of Economics, unpublished $\mathrm{PhD}$ thesis.

_ (2005), 'The Upward Path to Structural Realism', Philosophy of Science, vol. 72(5): 1361-1372.

- (2007), 'Uninterpreted Equations and the Structure-Nature Distinction', Philosophical Inquiry, vol. 29(1-2): 57-71.

Worrall, John (1989), 'Structural Realism: The Best of Both Worlds?' in Papineau, D. (ed.) The Philosophy of Science, Oxford: Oxford University Press, 1996.

Worrall, John and Elie Zahar (2001), 'Ramseyfication and Structural Realism', Appendix IV in E. Zahar, Poincaré's Philosophy: From Conventionalism to Phenomenology, Chicago and La Salle (IL): Open Court.

Zahar, Elie (2001), Poincaré's Philosophy: From Conventionalism to Phenomenology, Chicago and La Salle (IL): Open Court. 